\title{
The Korean guideline for cervical cancer screening
}

\section{Kyung-Jin Min ${ }^{1, *}$, Yoon Jae Lee ${ }^{2, *}$, Mina Suh ${ }^{3}$, Chong Woo Yoo ${ }^{4}$, Myong Cheol Lim ${ }^{5}$, Jaekyung Choi ${ }^{6}$, Moran Ki ${ }^{7}$, Yong-Man Kim ${ }^{8}$, Jae-Weon Kim ${ }^{9}$, Jea-Hoon Kim ${ }^{10}$, Eal Whan Park ${ }^{11}$, Hoo-Yeon Lee ${ }^{12}$, Sung-Chul Lim ${ }^{13}$, Chi-Heum $\mathrm{Cho}^{14}$, Sung Ran Hong ${ }^{15}$, Ji Yeon Dang ${ }^{3}$, Soo Young Kim ${ }^{16}$, Yeol Kim ${ }^{3}$, Won-Chul Lee ${ }^{17}$, Jae-Kwan Lee}

${ }^{1}$ Department of Obstetrics and Gynecology, Korea University Medical Center, Seoul; ${ }^{2}$ Department of Korean Gynecology, Jaseng Hospital of Korean Medicine, Seoul; ${ }^{3}$ National Cancer Control Institute, National Cancer Center, Goyang; ${ }^{4}$ Center for Uterine Cancer and Department of Pathology, National Cancer Center, Goyang; ${ }^{5}$ Center for Uterine Cancer, Hospital, Gynecologic Cancer Branch, Research Institute, Department of Cancer Control and Policy, Graduate School of Cancer Science and Policy, National Cancer Center, Goyang; ${ }^{6}$ Department of Family Medicine, Konkuk University Medical Center, Konkuk University School of Medicine, Seoul; 'Department of Cancer Control and Policy, Graduate School of Cancer Science and Policy, National Cancer Center, Goyang; ${ }^{8}$ Department of Obstetrics and Gynecology, Asan Medical Center, University of Ulsan College of Medicine, Seoul; ${ }^{9}$ Department of Obstetrics and Gynecology, Seoul National University Hospital, Seoul National University College of Medicine, Seoul; ${ }^{10}$ Department of Obstetrics and Gynecology, Gangnam Severance Hospital, Yonsei University College of Medicine, Seoul; Departments of ${ }^{11}$ Family Medicine, ${ }^{12}$ Social Medicine, Dankook University College of Medicine, Cheonan; ${ }^{13}$ Department of Pathology, Chosun University School of Medicine, Gwangju; ${ }^{14}$ Department of Obstetrics and Gynecology, Keimyung University School of Medicine, Daegu; ${ }^{15}$ Department of Pathology, Cheil General Hospital \& Women's Healthcare Center, Dankook University College of Medicine, Seoul; ${ }^{16}$ Department of Family Medicine, Hallym University Kangdong Sacred Heart Hospital, Hallym University College of Medicine, Seoul; ${ }^{17}$ Department of Preventive Medicine, The Catholic University of Korea College of Medicine, Seoul, Korea

The incidence rate of cervical cancer in Korea is still higher than in other developed countries, notwithstanding the national mass-screening program. Furthermore, a new method has been introduced in cervical cancer screening. Therefore, the committee for cervical cancer screening in Korea updated the recommendation statement established in 2002. The new version of the guideline was developed by the committee using evidence-based methods. The committee reviewed the evidence for the benefits and harms of the Papanicolaou test, liquid-based cytology, and human papillomavirus (HPV) testing, and reached conclusions after deliberation. The committee recommends screening for cervical cancer with cytology (Papanicolaou test or liquid-based cytology) every three years in women older than 20 years of age (recommendation A). The cervical cytology combined with HPV test is optionally recommended after taking into consideration individual risk or preference (recommendation C). The current evidence for primary HPV screening is insufficient to assess the benefits and harms of cervical cancer screening (recommendation I). Cervical cancer screening can be terminated at the age of 74 years if more than three consecutive negative cytology reports have been confirmed within 10 years (recommendation D).

Keywords: Mass Screening; Papanicolaou Test; Uterine Cervical Neoplasms

Received Jun 15, 2015, Revised Jun 26, 2015, Accepted Jun 26, 2015

\footnotetext{
*The first two authors contributed equally to this study.

*This article is a secondary publication of the guideline published in Journal of the Korean Medical Association in May 2015. Please cite the original article as: Min KJ, Lee YJ, Suh M, Yoo CW, Lim MC, Choi J, Ki M, Kim YM, Kim JW, Kim JH, Park EW, Lee HY, Lim SC, Cho CH, Hong SR, Dang JY, Kim SY, Kim Y, Lee WC, Lee JK. The Korean guideline for cervical cancer screening. J Korean Med Assoc. 2015 May;58(5):398-407. @ Korean Medical Association
}

Correspondence to Jae-Kwan Lee

Department of Obstetrics and Gynecology, Korea University Medical Center, Korea University College of Medicine, 148 Gurodong-ro, Guro-gu, Seoul 152703, Korea. E-mail: jklee38@gmail.com 


\section{EVIDENCE OF SCREENING AND LEVEL OF EVIDENCE}

1. The effect of cervical cancer screening using Pap smear or liquid-based cytology test is considered to have moderate level of evidence and evaluated to be substantially more beneficial than harmful for screening.

2. The evidence for benefits and harms of exclusive HPV test for cervical cancer screening is very low.

3. The level of evidence for screening effect of the cervical cytology combined with HPV test is moderate. The combined test is a little more beneficial than the exclusive cervical cytology.

\section{SCREENING GUIDELINE AND RECOMMENDATION GRADE}

1. This guideline recommends screening of asymptomatic women aged over 20 with Pap smear or liquid-based cytology every 3 years (recommendation $\mathrm{A}$ ).

2. The HPV test along with cervical cytology (Pap smear or liquidbased cytology) is optionally recommended in consideration of clinical decision for individual risk and preference of examinee (recommendation C).

3. The evidence for exclusive HPV test is insufficient to assess benefits and harms of cervical cancer screening (recommendation I).

4. Cervical cancer screening can be terminated at the age of 74 after three consecutive negative results of cervical cancer screening within 10 years (recommendation $\mathrm{D}$ ).

\section{RECOMMENDATION FOR SPECIAL CONSIDERATION}

1. Cervical cancer screening is not recommended for women who underwent total hysterectomy and had no past history of CIN2 or more lesions.

2. This guideline recommends continuous cervical cancer screening regardless of HPV vaccination.

3. This guideline recommends continuous cervical cancer screening regardless of pregnancy.

\section{BENEFITS AND HARMS OF SCREENING}

\section{Benefits of screening}

In cervical cancer, precancerous lesion such as CIN is well known. Therefore adequate treatment can be performed using relatively simple surgery such as conization and hence cervical cancer screening has benefit for preventing the development of cervical cancer. Cervical cancer screening using Pap smear decreased the incidence rate of cervical cancer by $62 \%$ in cohort study and $65 \%$ in case-control group study. The Korean studies also showed that cervical cancer screening using Pap smear reduced $64 \%$ of risk of mortality. The combination of HPV test with cervical cytology has further reduced the incidence rate of cervical cancer compared to the exclusive cervical cytology.

\section{Harms of screening}

There can be harms of cervical cancer screening such as overdiagnosis or side effects of diagnostic test due to false-positivity. Short-term psychological harms can also occur in case of HPV DNA test.

\section{CONSIDERATIONS IN CLINICAL PRACTICE}

1. This guideline is intended for asymptomatic women aged over 20.

2. Women belonging to high risk group with clinical symptoms such as irregular uterine bleeding, pain, or clinician's decision require adequate screening tests according to clinician's decision.

3. If a woman is diagnosed as CIN2 or more, termination time for screening should be determined based on clinician's decision.

4. Screening interval for cervical cancer can be longer than 3 years when a combined test is performed.

\section{INTRODUCTION}

In Korea, age-standardized incidence rate of cervical cancer has been steadily decreasing with the rate of 18.6 per 100,000 in 1999 to 11.7 per 100,000 in 2011 [1]. As of 2012, the agestandardized incidence rate of cervical cancer in Korea was lower than that of Japan whereas higher than that of other developed countries such as United States or United Kingdom [1]. Moreover, according to the National Cancer Registry statistics in 2012, the survival rate of cervical cancer was high compared to other cancers with the 5-year survival rate of $80.1 \%$ from 2007 to 2011 [1]. It was estimated that 4 of 5 people would survive more than 5 years after their initial diagnosis. One of the substantial factors that led to this critical change is the National Cancer Screening Program in Korea. Human papillomavirus (HPV) infection was found to play a crucial role in cause of cervical cancer and HPV test is being presented as a new screening method for cervical cancer; therefore, the review on cervical cancer screening guideline was necessary.

Moreover, the age distribution of cervical cancer is changing and studies regarding appropriate commencing age of screening are being reported. Consequently new screening guidelines including adjusted timing of cervical cancer screening are being published in multiple nations and organizations [2-10]. To cope with such changes, a novel cervical cancer screening guideline that meets the situation in Korea is needed. We evaluated the effectiveness of the National Cancer Screening Program and systemically reviewed the 
recent related literature and guidelines.

The purpose of this guideline is to develop evidence-based cervical cancer screening guideline for physicians and to provide information on benefits and harms of screening to public.

\section{DEVELOPMENT METHOD}

\section{Organization of committee and selection of key ques-} tions

To develop an evidence-based cervical cancer screening guideline, 'National Cervical Cancer Screening Guideline Development Committee' was constituted of experts recommended by the Korean Society of Gynecologic Oncology, the Korean Society for Cytopathology, the Korean Society for Preventive Medicine, and the Korean Academy of Family
Medicine. The committee evaluated scientific evidence relevant to the efficacy of cervical cancer screening through systematic review of related guidelines and literatures.

The committee formulated key questions to evaluate the effectiveness such as reduction in mortality, decreased incidence, and the harms of cervical cancer screening. The committee also formulated key questions to develop guidelines regarding the screening method to choose, commencing age, termination age, and interval of screening.

Selected key questions are shown in Table 1 and analytic framework in Fig. 1.

\section{Literature search and quality assessment}

We firstly searched previously published evidence-based cervical cancer screening guideline from National Guideline Clearing House, PubMed, and Guidelines International Network by using 'cervical cancer screening guideline' as key-

Table 1. Selected key questions by National cervical cancer screening guideline development committee

Key question 1: Does cervical cancer screening with cervical cytology reduce the mortality rate, the ratio of advanced cancer and the incidence of cervical cancer?

Key question 2: What are the harms of false-positive of cervical cancer screening using cervical cytology? Is the harms related to falsepositive of cervical cancer screening smaller than benefits of screening?

- Key question 3: What is the accuracy of liquid-based cytology compared with Pap smear? Does the harms such as insufficient samples occurs more in liquid-based cytology?

- Key question 4: Do cervical cancer screening of only human papillomavirus (HPV) test and combined test of cervical cytology and HPV test reduce the mortality rate, the ratio of advanced cancer and the incidence of cervical cancer?

Key question 5: What are the false-positive and resulting the psychological harms in screening using HPV test?

Key question 6: What is the age to begin, the age to terminate and screening interval?

Key question 7: The hysterectomized, HPV-vaccinated or pregnant women should equally implement routine cervical cancer screening?

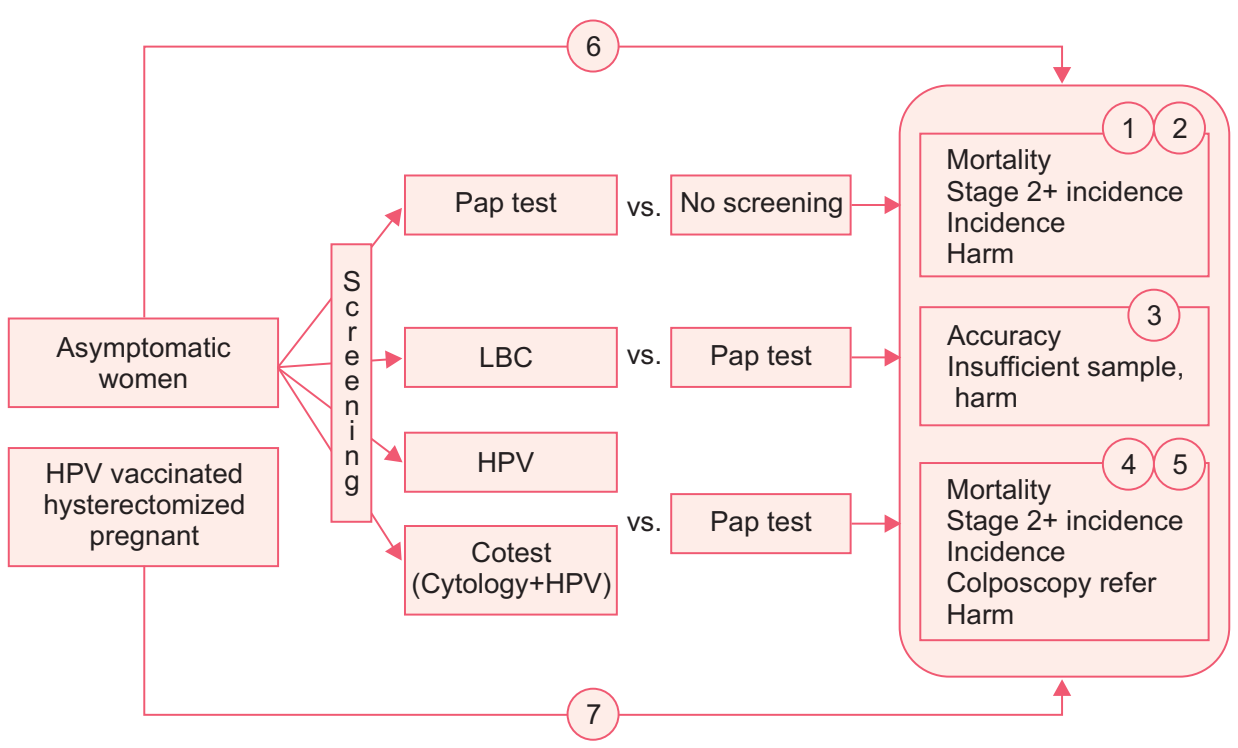

Fig. 1. Framework of developing a guideline for cervical cancer screening. (1) Benefits of pap test screening, (2) harms of pap test screening, (3) accuracy and harm of liquid based cytology (LBC), (4) benefits of human papillomavirus (HPV) primary test or cotest, (5) harms of HPV primary test or cotest, (6) target age and interval of cervical cancer screening, and (7) specific population group for cervical cancer. 
words to check guidelines subjected to adaptation. We also reviewed related evidences described in other guidelines and considered whether to adapt previously published guidelines or accept for the Korean guideline for cervical cancer screening.

In order to find the accordance with the selected key questions for benefits and harms of each screening methods, we searched international databases such as Medline, Cochrane Library as well as domestic databases: National Digital Science Library (NDSL), Korean Studies Information Service System (KISS), KoreaMed, Korea Institute of Science and Technology Information (KISTI), and Korean Medical Database (KMbase).

Selection and exclusion of literatures searched for evidence of key questions was carried out independently by two reviewers per literature.

Quality assessment was performed with AGREE II (advancing guideline development, reporting and evaluation in health care) on searched guidelines [11], the Cochrane Collaboration's tool for assessing risk of bias on randomized control trial (RCT), and RoBANS (risk of bias assessment tool for nonrandomized study) for non-randomized-control trial or observational study [12]. And AMSTAR (a measurement tool to assess the methodological quality of systematic reviews) was used for assessment of systematic review. In case of harmsrelated literatures [13], studies with no control groups were assessed by US Preventive Services Task Force because they cannot be assessed by RoBANS.

\section{Grade of recommendations and evidence}

The level of evidence from selected literatures for each key question was assessed by GRADE (the grading of recommendation, assessment, development and evaluation) [14]. In GRADE, the level of evidence is initially determined by study design. The level of evidence was classified as 'high' in case of
RCTs, and observational studies as 'low'. We downgraded the level of evidence by a level or two if there were any of risk of bias, inconsistency, indirectness, imprecision, or publication bias. Large effect, the effect of plausible confounding factors and dose-response gradient were considered as factors for upgrading level of evidence.

Final grade of evidence for cervical cancer screening was decided using the recommendation grading methods suggested by National Cancer Screening Program Revising Committee according to the level of recommendation evaluated by GRADE and the balance between benefits and harms of screening assessed by the committee.

\section{RESULTS}

\section{Results of literature search}

Five hundred and three cervical cancer screening related records were searched and 9 of 503 literatures were evidencebased screening guidelines [2-10]. Among them, eight guidelines were subjected to adaptation and one literature received low score in rigor of development by AGREE II [2-9]. We put evidence together through extracting the basis of evidence in guidelines and searching for additional latest literatures including domestic literatures for key questions (Fig. 2).

\section{Summary of evidence for each key questions and Recommendation Grade \\ 1) Key question 1: Does cervical cancer screening with cervical cytology reduce the mortality rate, the ratio of advanced cancer and the incidence of cervical cancer?}

Cervical cancer screening is a historic screening which has proven the effect of screening with many epidemiological data. However, it has been difficult to review the effect of

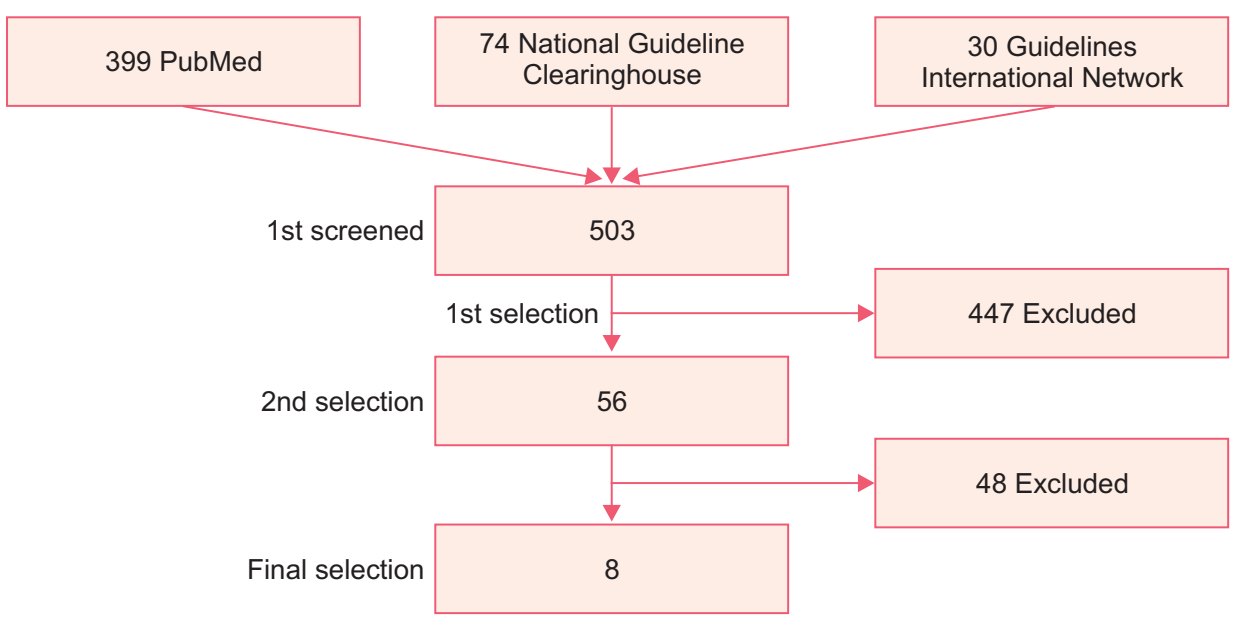

Fig. 2. Flow of guideline searching. 
screening since comparing RCT with no screening is currently not available.

In cervical cytology, the effectiveness of screening was evaluated based on the reduction of the mortality due to cervical cancer, the occurrence of advanced cancer and the incidence of cervical cancer. We confirmed the related evidence from one RCT, one cohort study, 12 case control studies, and two domestic studies [15-30]. Among them, one RCT was conducted in India and the effect of single screening from women with no cervical cancer screening experience was confirmed. However, the committee decided not to apply the result of the study as it is not suitable to situation in Korea due to differences in treatment for findings such as precancerous lesions identified by cervical cancer screening.

The reduction effect of cervical cancer mortality by Pap smear was reported in several epidemiological studies. Notably, the analysis of the recent Korean national cancer screening data confirmed the mortality reduction by cervical cancer screening as odds ratio 0.36 ( $95 \% \mathrm{Cl}, 0.31$ to 0.43$)$. According to a cohort study, the relative risk for the occurrence of cervical cancer decreased $62 \%$ by screening. In cervical cancer screening group from meta-analysis including 12 case control studies, the occurrence of cervical cancer decreased $65 \%$.

Based on these evidences, cervical cancer screening using Pap smear is evaluated as moderate level of evidence.

\section{2) Key question 2: What are the harms of false-positive} cervical cancer screening using cervical cytology? Are the harms related to false-positive cervical cancer screening smaller than benefits?

The false-positive cervical cytology may cause additional procedures such as colposcopy or colposcopy-directed punch biopsy which could lead to their side effects such as vaginal bleeding, pain, infection, or discharge. However, the side effects of colposcopy are rare and manageable even if they happen. Therefore the harm of false-positive cervical cytology is considered as small, and the benefit of cervical cytology is evaluated as "substantial." It is recommended as grade A.

\section{3) Key question 3: What is the accuracy of liquid-based} cytology compared to Pap smear? Does the harm such as insufficient samples occur more in liquid-based cytology?

The accuracy of liquid-based cytology was compared to that of Pap smear in consideration of the similarity of two examinations. The relative detection rate, sensitivity and specificity between liquid-based cytology and Pap smear that were compared and confirmed in RCT, as well as the accuracy from a cohort study were checked [31-35]. There were slight differences between studies; however, the accuracy of liquid-based cytology was considered similar to that of Pap smear. The committee decided to consider the level of evidence for the effect of liquid-based cytology as same as that of Pap smear due to their identical principle of examination. The falsepositive rate of liquid-based cytology was also similar to that of Pap smear while the rate of unsatisfactory sample by liquidbased cytology was lower than Pap smear in RCT. In addition, liquid-based cytology showed no additional direct harms in sample collection when compared to Pap smear. Therefore, liquid-based cytology is evaluated to have moderate level of evidence in accordance with Pap smear and consequently the benefit of screening is "substantial."

\section{4) Key question 4: Do cervical cancer screening of exclusive HPV test and the combined test of cervical cytology with $\mathrm{HPV}$ test reduce the mortality rate, the ratio of advanced cancer, and the incidence of cervical cancer compare to the exclusive cervical cytology?}

\section{5) Key question 5: What are the false-positivity and conse- quent psychological harms in screening using HPV test?}

In case of HPV test, the exclusive HPV test and the combined test of cervical cytology with HPV test were compared to check whether the mortality, stage 2 or more, or the incidence rate is reduced. We searched the reported evidence of exclusive HPV test reducing the incidence of cervical cancer when compared to cervical cytology. There was a result reported from one RCT, yet no significant effect was found and the number of related reports was not sufficient [36].

We tried to evaluate the harms of false-positivity by comparing cumulative colposcopy referral on regular repetitive screening. However, the relevant data was not available. The evidence for exclusive HPV test was considered insufficient by GRADE evaluation and hence graded as grade I.

The combined test of cervical cytology with HPV test was confirmed to have moderate level of evidence on reducing the incidence of cervical cancer in meta-analysis from four RCT [36-39]. However, the cumulative colposcopy referral due to false-positivity was analyzed to be escalated when compared to exclusive cervical cytology. The potential of psychological harms due to increase of false-positivity was considered in this guideline, yet evaluated as temporary harms due to its disappearance within 6 months. Consequently, the benefit of the combination test is "small" due to the harms of cumulative colposcopy referrals.

Therefore the committee decided that combined test of cervical cytology with HPV test is optionally recommended after taking into consideration of individual risk or preference. 


\section{6) Key question 6: What is the age to begin, the age to terminate, and the interval of screening?}

The evidence related to commencing age, terminating age, and screening interval, especially Korean evidence, was not sufficient. Therefore we considered epidemiological factors as an alternative. Since National Cancer Screening Guideline of 2002 for five major cancers, which suggested age to begin cervical cancer screening at age of 20, the incidence of cervical cancer has steadily decreased in Korea.

However, the incidence of cervical cancer is still higher than that of developed countries and the incidence of cervical carcinoma in situ or more has been steadily increasing in women of 20 s of age. Consequently, the committee recommended sustaining the age to begin screening as 20 to reduce the incidence of cervical cancer and to reduce complications of precancerous lesion treatment during pregnancy.

Unlike other countries, the incidence and mortality of cervical cancer tends to increase with age in Korea. Therefore the committee considered increasing the age to terminate screening more than other diseases. As a result, 74 was determined as age to terminate cervical cancer screening reflecting longer expected life span. However cervical cancer is known to occur after 15 to 25 years of HPV infection. Therefore the screening termination has to be limited to women who have three consecutive negative cytology within last 10 years and have no history of CIN2 or more within last 20 years.

According to analysis of domestic reports, cervical cytology with interval of 2 to 3 years was effective. The committee has decided to recommend Pap smear every 3 years regarding the cost-effectiveness of cervical cancer screening.

\section{7) Key question $7:$ Should the hysterectomized, HPV-vaccinated or pregnant women be equally implemented into routine cervical cancer screening?}

Guideline regarding post-hysterectomy, HPV-vaccinated women and other various clinical situations was difficult to find from literatures selected. Cervical cancer screening was not recommended for women who underwent hysterectomy and women with no history of CIN2 or more. In addition, since there is no sufficient evidence for the relationship between cervical cancer occurrence and HPV vaccination, cervical cancer screening is recommended regardless of HPV vaccination and pregnancy status as well.

\section{DISCUSSION}

We constituted a committee comprising multidisciplinary experts to reflect the latest trends and evidences and com- posed key questions. The committee systemically reviewed the existing guidelines and literatures to determine the effectiveness of cervical cancer screening.

This guideline is distinctive in several aspects compared to other ones. On the contrary to other guidelines which recommends both exclusive cervical cytology and combined cytology with HPV test, this guideline recommends either Pap smear or liquid-based cytology as grade A and combination with HPV test is optionally permitted according to the decision of clinician in consideration of the individual risk or preference of examinee. It is because of the assessment that harms of combined test by false-positivity occur even if it is effective for reducing the incidence of cervical cancer more than the exclusive cytology, and hence that the benefits of the combined test do not much exceed harms of it. In addition, considering the inconsistency in accuracy of HPV test, it was intensively discussed in the process of guideline development whether to recommend HPV test. Several HPV test of Korea that report multiple subtypes of HPV have been identified to be inconsistent while the tests for high-risk types of 16 and 18 have shown reproducible and reliable results, which led to the inclusion of HPV test into the guidelines. This reflects the view that the changes in management policy and result reporting of HPV tests are warranted.

In April 2014, an HPV DNA test was approved as a primary screening method by Food and Drug Administration; however, it was not clear if the HPV test reduces the incidence of cervical cancer compared to cervical cytology and if the harms from false-positivity increase. Further investigation is needed regarding the effect of exclusive HPV test and to determine if the harms from false-positivity increase. The reassessment of benefits and harms of exclusive HPV DNA screening is warranted.

In addition, the commencing and terminating age are different from other guidelines because the incidence and mortality of cervical cancer increase in old age in Korea contrary to other countries and the expected life span is longer. We believe setting the terminating age of screening to 74 will help to decrease the incidence of cervical cancer. The interval of screening is recommended as every 3 years with consideration of epidemiologic data, modeling study, and cost-effectiveness analysis. However, consideration is required regarding the analysis of cost-effectiveness implemented in this guideline because the prices of Pap smear and colposcopy are arranged quite low in Korea despite constant quality control and high accuracy.

The government henceforth is expected to establish policies of HPV DNA test such as quality assessment and to undertake related studies. Continuous revision of cervical cancer screening guidelines is warranted. 


\section{CONCLUSIONS}

This guideline recommends asymptomatic women aged over 20 to begin cervical cancer screening with Pap smear or liquid-based cytology every 3 years until the age of 74 when three-consecutive cytology show negative results within last ten years.

The combined test of cervical cytology with HPV DNA test is alternatively recommended after taking into account clinical decision and individual risk or preference. This guideline can be applied according to clinicians' decision and the age to terminate cervical cancer screening for women with history of CIN2 or more should be clinically determined.

\section{CONFLICT OF INTEREST}

No potential conflict of interest relevant to this article was reported.

\section{ACKNOWLEDGMENTS}

This study was supported by National Cancer Center Grant (1310870-2).

\section{REFERENCES}

1. National Cancer Control Institute. National Cancer Incidence 2012, Korea. Goyang: Ministry of Health and Welfare; 2014.

2. Moyer VA; U.S. Preventive Services Task Force. Screening for cervical cancer: U.S. Preventive Services Task Force recommendation statement. Ann Intern Med 2012;156:880-91, W312.

3. Canadian Task Force on Preventive Health Care, Dickinson J, Tsakonas E, Conner Gorber S, Lewin G, Shaw E, et al. Recommendations on screening for cervical cancer. CMAJ 2013;185:35-45.

4. Lee JK, Hong JH, Kang S, Kim DY, Kim BG, Kim SH, et al. Practice guidelines for the early detection of cervical cancer in Korea: Korean Society of Gynecologic Oncology and the Korean Society for Cytopathology 2012 edition. J Gynecol Oncol 2013;24:186-203.

5. Saslow D, Solomon D, Lawson HW, Killackey M, Kulasingam SL, Cain J, et al. American Cancer Society, American Society for Colposcopy and Cervical Pathology, and American Society for Clinical Pathology screening guidelines for the prevention and early detection of cervical cancer. CA Cancer J Clin 2012;62:147-72.

6. Committee on Practice Bulletins-Gynecology. ACOG Practice Bulletin Number 131: screening for cervical cancer. Obstet Gynecol 2012;120:1222-38.

7. Murphy J, Kennedy EB, Dunn S, McLachlin CM, Fung Kee Fung M, Gzik D, et al. Cervical screening: a guideline for clinical practice in
Ontario. J Obstet Gynaecol Can 2012;34:453-8.

8. Partridge EE, Abu-Rustum NR, Campos SM, Fahey PJ, Farmer M, Garcia RL, et al. Cervical cancer screening. J Natl Compr Canc Netw 2010;8:1358-86.

9. Hamashima C, Aoki D, Miyagi E, Saito E, Nakayama T, Sagawa M, et al. The Japanese guideline for cervical cancer screening. Jpn J Clin Oncol 2010;40:485-502.

10. Royal Australian College of General Practitioners. Early detection of cancers. In: Guidelines for preventive activities in general practice. 8th ed. East Melbourne: Royal Australian College of General Practitioners; 2012. p. 60-72.

11. Brouwers MC, Kho ME, Browman GP, Burgers JS, Cluzeau F, Feder G, et al. AGREE II: advancing guideline development, reporting and evaluation in health care. CMAJ 2010;182:E839-42.

12. Kim SY, Park JE, Lee YJ, Seo HJ, Sheen SS, Hahn S, et al. Testing a tool for assessing the risk of bias for nonrandomized studies showed moderate reliability and promising validity. J Clin Epidemiol 2013; 66:408-14.

13. Shea BJ, Hamel C, Wells GA, Bouter LM, Kristjansson E, Grimshaw $J$, et al. AMSTAR is a reliable and valid measurement tool to assess the methodological quality of systematic reviews. J Clin Epidemiol 2009;62:1013-20.

14. Guyatt G, Oxman AD, Akl EA, Kunz R, Vist G, Brozek J, et al. GRADE guidelines: 1. Introduction-GRADE evidence profiles and summary of findings tables. J Clin Epidemiol 2011;64:383-94.

15. Sankaranarayanan R, Nene BM, Shastri SS, Jayant K, Muwonge R, Budukh AM, et al. HPV screening for cervical cancer in rural India. N Engl J Med 2009;360:1385-94.

16. Herbert A, Stein K, Bryant TN, Breen C, Old P. Relation between the incidence of invasive cervical cancer and the screening interval: is a five year interval too long? J Med Screen 1996;3:140-5.

17. Andrae B, Kemetli L, Sparen P, Silfverdal L, Strander B, Ryd W, et al. Screening-preventable cervical cancer risks: evidence from a nationwide audit in Sweden. J Natl Cancer Inst 2008;100:622-9.

18. Aristizabal N, Cuello C, Correa P, Collazos T, Haenszel W. The impact of vaginal cytology on cervical cancer risks in Cali, Colombia. Int J Cancer 1984;34:5-9.

19. Berrino F, Gatta G, d'Alto M, Crosignani P, Riboli E. Efficacy of screening in preventing invasive cervical cancer: a case-control study in Milan, Italy. IARC Sci Publ 1986;(76):111-23.

20. Clarke EA, Anderson TW. Does screening by "Pap" smears help prevent cervical cancer? A case-control study. Lancet 1979;2:1-4.

21. Decker K, Demers A, Chateau D, Musto G, Nugent Z, Lotocki R, et al. Papanicolaou test utilization and frequency of screening opportunities among women diagnosed with cervical cancer. Open Med 2009:3:e140-7.

22. Hernandez-Avila M, Lazcano-Ponce EC, de Ruiz PA, Romieu I. Evaluation of the cervical cancer screening programme in Mexico: a population-based case-control study. Int J Epidemiol 1998;27:370-6.

23. Herrero R, Brinton LA, Reeves WC, Brenes MM, de Britton RC, Gaitan $E$, et al. Screening for cervical cancer in Latin America: a casecontrol study. Int J Epidemiol 1992;21:1050-6.

24. Hoffman M, Cooper D, Carrara H, Rosenberg L, Kelly J, Stander I, et 
al. Limited Pap screening associated with reduced risk of cervical cancer in South Africa. Int J Epidemiol 2003;32:573-7.

25. Jimenez-Perez M, Thomas DB. Has the use of pap smears reduced the risk of invasive cervical cancer in Guadalajara, Mexico? Int J Cancer 1999;82:804-9.

26. Makino H, Sato S, Yajima A, Komatsu S, Fukao A. Evaluation of the effectiveness of cervical cancer screening: a case-control study in Miyagi, Japan. Tohoku J Exp Med 1995;175:171-8.

27. Nieminen P, Kallio M, Anttila A, Hakama M. Organised vs. spontaneous Pap-smear screening for cervical cancer: a casecontrol study. Int J Cancer 1999;83:55-8.

28. Talbott EO, Norman SA, Kuller LH, Ishii EK, Baffone KM, Dunn MS, et al. Refining preventive strategies for invasive cervical cancer: a population-based case-control study. J Womens Health 1995;4:38795.

29. Cho BR. Evaluation of the validity of current national health screening program and plan to improve the system. Cheonju: Korea Centers for Disease Control and Prevention; 2013.

30. Jun JK, Choi KS, Jung KW, Lee HY, Gapstur SM, Park EC, et al. Effectiveness of an organized cervical cancer screening program in Korea: results from a cohort study. Int J Cancer 2009;124:188-93.

31. Siebers AG, Klinkhamer PJ, Grefte JM, Massuger LF, Vedder JE, Beijers-Broos A, et al. Comparison of liquid-based cytology with conventional cytology for detection of cervical cancer precursors: a randomized controlled trial. JAMA 2009;302:1757-64.

32. Ronco G, Cuzick J, Pierotti P, Cariaggi MP, Dalla Palma P, Naldoni $C$, et al. Accuracy of liquid based versus conventional cytology: overall results of new technologies for cervical cancer screening: randomised controlled trial. BMJ 2007;335:28.
33. Taylor S, Kuhn L, Dupree W, Denny L, De Souza M, Wright TC Jr. Direct comparison of liquid-based and conventional cytology in a South African screening trial. Int J Cancer 2006;118:957-62.

34. Coste J, Cochand-Priollet B, de Cremoux P, Le Gales C, Cartier I, Molinie $\mathrm{V}$, et al. Cross sectional study of conventional cervical smear, monolayer cytology, and human papillomavirus DNA testing for cervical cancer screening. BMJ 2003;326:733.

35. Ko MJ, Kim Y, Hong SR, Lee JK, Shim J, Kim J, et al. Cost-effectiveness of conventional cytology and HPV DNA testing for cervical cancer screening in South Korea. Seoul: National Evidence-based Healthcare Collaborating Agency; 2014.

36. Ronco G, Giorgi-Rossi P, Carozzi F, Confortini M, Dalla Palma P, Del Mistro A, et al. Efficacy of human papillomavirus testing for the detection of invasive cervical cancers and cervical intraepithelial neoplasia: a randomised controlled trial. Lancet Oncol 2010;11:24957.

37. Bulkmans NW, Berkhof J, Rozendaal L, van Kemenade FJ, Boeke AJ, Bulk S, et al. Human papillomavirus DNA testing for the detection of cervical intraepithelial neoplasia grade 3 and cancer: 5-year follow-up of a randomised controlled implementation trial. Lancet 2007;370:1764-72.

38. Naucler P, Ryd W, Tornberg S, Strand A, Wadell G, Elfgren K, et al. Human papillomavirus and Papanicolaou tests to screen for cervical cancer. N Engl J Med 2007;357:1589-97.

39. Kitchener HC, Almonte M, Thomson C, Wheeler P, Sargent A, Stoykova $B$, et al. HPV testing in combination with liquid-based cytology in primary cervical screening (ARTISTIC): a randomised controlled trial. Lancet Oncol 2009;10:672-82. 